\title{
Outdoor Robot Navigation Based on a Probabilistic Data Fusion Scheme
}

\author{
Josep M. Mirats Tur and Carlos Albores Borja
}

\begin{abstract}
This article presents a data fusion method which seeks to obtain better pose estimation of a mobile robot through obtaining a more accurate covariance uncertainty matrix. We seek to compute the state covariance without using the first-order linear approximations of the extended Kalman filter. We consider, unlike standard work done in error propagation and data fusion, the possible correlation between the different sensor pose estimates, odometry and DGPS for the present work, and the autocorrelation of some of the variables involved in the fusion (DGPS data, for the particular case herein presented). Computation of the covariances of each sensor data vector is presented so it takes into account the vehicle kinematics, and hence, its particular characteristics. In order to validate the presented approach, a real outdoor navigation experiment is presented fusing odometry and DGPS data.
\end{abstract}

Index Terms - Data fusion, uncertainty position estimation, robot localization, robot navigation.

\section{INTRODUCTION}

$\mathrm{M}$ OBILE robots have generated great interest in recent years due to their capacity to work in remote or harmful environments where humans cannot enter because of the extreme operating conditions [1]-[3]. Several applications are found in the literature, varying from material transportation in a common industrial environment, [4], to the exciting exploration of a far planet surface [5].

In spite of the increasing number of applications on mobile robotics, there are still challenging research efforts to be done. The problem of localization and navigation has been widely studied [6]. It is necessary for a robot, in order to correctly perform the assigned task, to calculate its pose (position and orientation) in the environment. Whichever

Manuscript received Mar. 27th, 2007. This work has been supported by the Spanish Superior Council for Scientific Research (CSIC).

Josep M. Mirats Tur is actually working as a full time researcher in the Institut de Robòtica i Informàtica Industrial (IRI), Parc Tecnològic de Barcelona, Edifici U, C. Llorens i Artigas, 4-6, 2a planta, 08028 Barcelona, Spain (phone: +34 93 4054261; fax: +34 93 4015750; e-mail: jmirats@iri.upc.edu).

Carlos Albores is a $\mathrm{PhD}$ student in the Center for Intelligent Systems (CSI), Tecnológico de Monterrey (ITESM), $5^{\circ}$ piso torre sur CETEC, Av. Eugenio Garza Sada 2501, 64849 Monterrey N.L., México (e-mail: carlos.albores@itesm.mx). the sensory system used, the computation of the robot's pose estimate will never be error free: there is always an associated uncertainty to this estimate. Therefore, localization involves two problems: computing a position estimate and its associated uncertainty.

A common and basic pose estimation technique is dead reckoning or odometry [7, 8]. Unfortunately, the uncertainty of the robot pose grows as it moves, to such degree, that, after a relatively small period of time, it is no longer safe for the robot to perform a task, mainly, because it does not know where it is. High pose estimation errors lead to poor performances, and probable collisions. An adequate technique to reduce pose uncertainty is to use redundant data combining two or more data sources. The way in which the data of multiple sources are processed to obtain a better estimation is called Data fusion [9]. A widely used technique when fusing pose information is Kalman filtering, concretely the Extended Kalman Filter (EKF) [10], with the problem of determining the covariance matrices associated with the pose uncertainty. Different schemes for data fusion, tightly or loosely coupled, normally based on the EKF, are reported in [11].

Following our previous work presented in $[12,13]$ in which a method to determine the covariance matrices associated with the pose uncertainty is developed, and working on a data fusion technique based on probabilities reported in [14], which considers correlation between variables in its fusion system, we propose a probabilistic data fusion scheme extending the method in [14] with the covariance computation we proposed in $[12,13]$. Using this method, odometry and DGPS data are fused in order to obtain the pose and associated uncertainty of the robot presented in the appendix A.

So this paper is mainly focused on two issues: first, how to accurately measure the odometry pose uncertainty taking into consideration the particular characteristics of the used vehicle, and, afterwards, how to reduce the robot's pose uncertainty by means of fusing information from different sensors which do not necessarily provide independent data, but correlated among each other.

The paper is organized as follows: Section 2 revises the proposed error model; Section 3 describes the used data 
fusion technique; Section 4 contains results from data fusion experiments and comparison against the well known EKF, and, finally, Section 5 addresses conclusions and future work.

\section{ODOMETRY POSE UNCERTAINTY: COVARIANCE ESTIMATION}

A general way to obtain an expression for the pose uncertainty, by means of a covariance matrix, when robot pose estimates are being obtained using internal sensors was presented in a previous work, where a particularization was made for a non-holonomic Ackerman driven autonomous vehicle. This section summarizes the obtained results of this work. The particularization of these formulae to the robot used for the present study is handled in the Appendix B.

Summarizing the concepts given in [12] and [13], suppose that for time ${ }^{1} k-1$, the pose of the vehicle and its associated uncertainty are known, $P_{k-1}=\left\{\left[x_{k-1}, y_{k-1}, \theta_{k-1}\right]\right.$, $\left.\operatorname{cov}\left[P_{k-1}\right]\right\}$. Then, for time $k$, after the vehicle has performed a certain movement on the plane, and sensors on the robot have noisily measured this displacement, the pose of the vehicle is obtained using $P_{k}=P_{k-1}+\Delta P_{k}$, where $\Delta P_{k}$ $=\left[\Delta x_{k}, \Delta y_{k}, \Delta \theta_{k}\right]$. Uncertainty of the pose estimate is then given by the covariance $\operatorname{cov}\left[P_{k}\right]=\operatorname{cov}\left[P_{k-1}\right]+\operatorname{cov}\left[\Delta P_{k}\right]+\operatorname{cov}\left[P_{k-1}, \Delta P_{k}^{T}\right]+\operatorname{cov}\left[\Delta P_{k}, P_{k-1}^{T}\right] . \quad$ The term $\operatorname{cov}\left[P_{k-1}\right]$ is recursively defined and is initialized to $O_{3 \times 3}$ if the initial pose of the robot is well known. In our first work [12], we considered the influence of the previous position $P_{k-l}$, on the increment of run path $\Delta P_{k}$, as not meaningful, i.e., $\operatorname{cov}\left[P_{k-1}, \Delta P_{k}^{T}\right]+\operatorname{cov}\left[\Delta P_{k}, P_{k-1}^{T}\right]=0$. However, in a recent work [13], we have seen that this hypothesis is not valid in general and propose a further development of the cross-covariance terms. In order to compute them, an error vector for the robot's pose in time $k-1$ is given by, $\hat{P}_{k-1}=P_{k-1}+\varepsilon_{P_{k-1}}$ where $\varepsilon_{P_{k-1}}=\left[\begin{array}{lll}\varepsilon_{x_{k-1}} & \varepsilon_{y_{k-1}} & \varepsilon_{\theta_{k-1}}\end{array}\right]^{T}$. Now, since $\operatorname{Cov}\left(\hat{P}_{k-1}\right)$ is a positive semi definite Hermitian matrix, it could be decomposed in the form $\operatorname{Cov}\left(\hat{P}_{k-1}\right)=Q \lambda Q^{T}, \operatorname{cov}(\gamma)=\lambda$ where $Q$ is the eigenvector matrix and $\lambda$ is a diagonal matrix containing the eigenvalues of $\operatorname{Cov}\left(\hat{P}_{k-1}\right)$. The eigen-values could be seen as the covariance of independent, zero mean, Gaussian and orthogonal errors $\gamma$. So this term $Q \gamma$ is capable of modeling the rotation of the robot pose uncertainty as it runs its path, allowing the calculation of the crossed covariance terms. Finally, the general expression for the term $\operatorname{cov}\left[\Delta P_{k}\right]$ is

$$
\operatorname{cov}\left[\Delta P_{k}\right]=E\left[\Delta P_{k} \Delta P_{k}^{T}\right]-E\left[\Delta P_{k}\right] E\left[\Delta P_{k}^{T}\right]
$$

\footnotetext{
${ }^{1}$ We are using time step $k-1$, for $t-\Delta t, k-2$ for $t-2 \Delta t$, and so on.
}

$$
\begin{gathered}
E\left[\Delta P_{k} \Delta P_{k}^{T}\right]=\left(\begin{array}{lll}
E\left[\Delta x_{k} \Delta x_{k}^{T}\right] & E\left[\Delta x_{k} \Delta y_{k}^{T}\right] & E\left[\Delta x_{k} \Delta \theta_{k}^{T}\right] \\
E\left[\Delta y_{k} \Delta x_{k}^{T}\right] & E\left[\Delta y_{k} \Delta y_{k}^{T}\right] & E\left[\Delta y_{k} \Delta \theta_{k}^{T}\right] \\
E\left[\Delta \theta_{k} \Delta x_{k}^{T}\right] & E\left[\Delta \theta_{k} \Delta y_{k}^{T}\right] & E\left[\Delta \theta_{k} \Delta \theta_{k}^{T}\right]
\end{array}\right) \\
E\left[\Delta P_{k}\right] E\left[\Delta P_{k}^{T}\right]=\left(\begin{array}{lll}
E\left[\Delta x_{k}\right] E\left[\Delta x_{k}^{T}\right] & E\left[\Delta x_{k}\right] E\left[\Delta y_{k}^{T}\right] & E\left[\Delta x_{k}\right] E\left[\Delta \theta_{k}^{T}\right] \\
E\left[\Delta y_{k}\right] E\left[\Delta x_{k}^{T}\right] & E\left[\Delta y_{k}\right] E\left[\Delta y_{k}^{T}\right] & E\left[\Delta y_{k}\right] E\left[\Delta \theta_{k}^{T}\right] \\
E\left[\Delta \theta_{k}\right] E\left[\Delta x_{k}^{T}\right] & E\left[\Delta \theta_{k}\right] E\left[\Delta y_{k}^{T}\right] & E\left[\Delta \theta_{k}\right] E\left[\Delta \theta_{k}^{T}\right]
\end{array}\right)
\end{gathered}
$$

\section{DATA FUSION}

Assume now, that we have several pose estimations, each one with its associated error, derived from different sensors carried on the robot but, obviously, computed with respect to the same reference system: $\left\{P_{k}^{s 1}, \operatorname{cov}\left[P_{k}^{s 1}\right]\right\}$ for sensor $1, \ldots,\left\{P_{k}^{s n}, \operatorname{cov}\left[P_{k}^{s n}\right]\right\}$ for sensor $n$. We want to calculate a new pose estimate, as well as its uncertainty, $\left\{P_{k}^{f}, \operatorname{cov}\left[P_{k}^{f}\right]\right\}$, using all the information from the various sensors.

In order to compute the probability of a given pose estimate we need to consider a particular probability distribution function. Without loss of generality, the following development is particularized to the system used in this study. Two pose estimates are available, one computed from odometry, $\left\{P_{k}^{o d}, \operatorname{cov}\left[P_{k}^{o d}\right]\right\}$, the other from a GPS receiver, $\left\{P_{k}^{g p s}, \operatorname{cov}\left[P_{k}^{G P S}\right]\right\}$. For both sensors, after systematic errors have been calibrated, normal distribution of the errors are assumed. Although odometry errors is assumed to be affected by white noise [15], meaning that they are not correlated with themselves, experimental data reveal that, as shown in Appendix C, GPS (or DGPS) pose estimates are autocorrelated, so its errors are affected by colored noise. By now, assume that each independent covariance matrix expressing the uncertainty in the pose estimates is known. The probabilities that the fused pose estimate, denoted by, $P_{k}^{f}$, takes whether to the $\left\{P_{k}^{o d}, \operatorname{cov}\left[P_{k}^{o d}\right]\right\}$ or the $\left\{P_{k}^{G P S}, \operatorname{cov}\left[P_{k}^{G P S}\right]\right\}$ are given by the normal probability distribution equation:

$$
\begin{aligned}
& \operatorname{Pr}^{o d}\left(P_{k}^{f}\right)=\frac{e^{-\frac{1}{2}\left(P_{k}^{f}-P_{k}^{o d}\right)^{T} \operatorname{cov}^{-1}\left[P_{k}^{o d}\right]\left(P_{k}^{f}-P_{k}^{o d}\right)}}{(2 \pi)^{\frac{3}{2}} \sqrt{\left|\operatorname{cov}^{-1}\left[P_{k}^{o d}\right]\right|}}(4) \\
& \operatorname{Pr}^{G P S}\left(P_{k}^{f}\right)=\frac{e^{-\frac{1}{2}\left(P_{k}^{f}-P_{k}^{G P S}\right)^{T} \operatorname{cov}^{-1}\left[P_{k}^{G P S}\right]\left(P_{k}^{f}-P_{k}^{G P S}\right)}}{(2 \pi)^{\frac{3}{2}} \sqrt{\left|\operatorname{cov}^{-1}\left[P_{k}^{G P S}\right]\right|}}
\end{aligned}
$$

For each time $k$, the best pose estimate $P_{k}^{f}$ is the one for which the probability of both distributions, corresponding to the joint probability distribution, is maximum. The joint probability distribution of two normal distributions is another normal distribution where $\operatorname{cov}_{k}^{\text {od-GPS }}$ holds the correlation between GPS and odometry: 


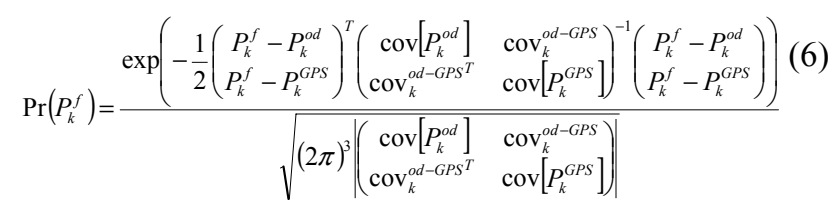

For the particular case in which the different pose estimates are independent, the joint probability distribution is expressed as the product of the independent distribution probabilities. This would simplify the process of pose fusion; unfortunately this is not the case. As odometers measure the relative displacement with respect to the previous pose, the computation of the odometry pose estimate, $P_{k}^{o d}$ depends on the fused pose at time $k-1: P_{k}^{o d}=$ $P_{k-1}^{f}+\Delta P_{k}^{o d}$ which in turn depends on the information gathered from the GPS. In order to express (6) as the product of two normal distributions it is necessary to transform its covariance matrix in a block diagonal matrix. This is done through Cholesky decomposition of the covariance matrix into a product of the kind:

$\left(\begin{array}{ll}A & B \\ B & C\end{array}\right)^{-1}=\left(\begin{array}{cc}I & 0 \\ -C^{-1} B^{T} & I\end{array}\right) \cdot\left(\begin{array}{cc}A-B C^{-1} B^{T} & 0 \\ 0 & C\end{array}\right)^{-1}\left(\begin{array}{cc}I & -C B^{-1} \\ 0 & I\end{array}\right)$

where $\mathrm{A}=\operatorname{cov}\left[P_{k}^{o d}\right], \mathrm{B}=\operatorname{cov}_{k}^{\text {od-GPS }}, \mathrm{C}=\operatorname{cov}\left[P_{k}^{G P S}\right], \mathrm{I}=\mathrm{I}_{3 \times 3}$, $0=0_{3 \times 3}$. Then, substituting (7) into (6) we obtain:

$\operatorname{Pr}\left(P_{k}^{f}\right)=\frac{\exp \left(-\frac{1}{2}\left(\begin{array}{c}P_{k}^{f}-\widetilde{P}_{k}^{o d} \\ P_{k}^{f}-P_{k}^{G P S}\end{array}\right)^{T}\left(\begin{array}{cc}\operatorname{cov}\left[\widetilde{P}_{k}^{o d}\right] & 0 \\ 0 & \operatorname{cov}\left[P_{k}^{G P S}\right]\end{array}\right)^{-1}\left(\begin{array}{c}P_{k}^{f}-\widetilde{P}_{k}^{o d} \\ P_{k}^{f}-P_{k}^{G P S}\end{array}\right)\right)}{\sqrt{(2 \pi)^{3}\left(\begin{array}{cc}\operatorname{cov}\left[\widetilde{P}_{k}^{o d}\right] & 0 \\ 0 & \operatorname{cov}\left[P_{k}^{G P S}\right]\end{array}\right) \mid}}$

where:

$\widetilde{P}_{k}^{o d}=P_{k}^{o d}+\operatorname{cov}_{k}^{o d-G P S} \operatorname{cov}^{-1}\left[P_{k}^{G P S}\right]\left(P_{k}^{f}-P_{k}^{G P S}\right)$

$\operatorname{cov}\left[\widetilde{P}_{k}^{o d}\right]=\operatorname{cov}\left[P_{k}^{o d}\right]-\operatorname{cov}_{k}^{o d-G P S} \operatorname{cov}^{-1}\left[P_{k}^{G P S}\right] \operatorname{cov}_{k}^{o d-G P S T}$

In order to obtain a fused pose estimate that maximizes the joint probability of equation (8), we differentiate with respect to $P_{k}^{f}$ and equal to zero the result. Finally, the obtained equations for the fused pose and its associated uncertainty, $\left\{P_{k}^{f}, \operatorname{cov}\left[P_{k}^{f}\right]\right\}$, are:

$P_{k}^{f}=P_{k}^{o d}+G_{k}\left(P_{k}^{G P S}-P_{k}^{o d}\right)$

where,

$G_{k}=\mathbf{I}-\left\{\left[\operatorname{cov}^{-1}\left[P_{k}^{G P S}\right]+M^{T} \operatorname{cov}^{-1}\left[\widetilde{P}_{k}^{o d}\right] M\right]^{-1} M^{T} \operatorname{cov}^{-1}\left[\widetilde{P}_{k}^{o d}\right]\right\}$

$M=\left(\mathbf{I}-\operatorname{cov}_{k}^{o d-G P S} \operatorname{cov}^{-1}\left[P_{k}^{G P S}\right]\right)$

The uncertainty of the fused pose given by its covariance matrix is:

$$
\begin{aligned}
& \operatorname{cov}\left[P_{k}^{f}\right]=\operatorname{cov}\left[P_{k}^{o d}\right]+G_{k}\left(\operatorname{cov}_{k}^{o d-G P S^{T}}-\operatorname{cov}\left[P_{k}^{o d}\right]\right)+ \\
& \cdot\left(\operatorname{cov}_{k}^{o d-G P S}-\operatorname{cov}\left[P_{k}^{o d}\right]\right) G_{k}^{T}+ \\
& \cdot G_{k}\left(\operatorname{cov}\left[P_{k}^{G P S}\right]-\operatorname{cov}_{k}^{o d-G P S^{T}}-\operatorname{cov}_{k}^{o d-G P S}+\operatorname{cov}\left[P_{k}^{o d}\right]\right) G_{k}{ }^{T}
\end{aligned}
$$

Please note that for the special case when no GPSodometry correlation is considered, i.e., $\operatorname{cov}_{k}^{o d-G P S}=0$, the presented formulation leads to the traditional Kalman filter.

\section{ASSESSMENT OF RESULTS}

In order to assess the validity of the proposed data fusion and covariance computation methods, with the main purpose of obtaining a pose estimate for the robot presented in Appendix A, an experiment was designed and performed in a parking lot in front of the IRI. We used for this experiment the data fusion framework presented in Section III together with the odometry pose uncertainty calculation explained in Section II. The experiment consisted on automatically following a $P$ figure path. Data from both, internal sensors (encoders), and DGPS sensor were gathered during the trajectory. We then computed the fused pose estimates, as well as their uncertainties, using the method presented in this paper, and using standard EKF.

Figure 1 shows the estimated trajectory when using only odometry data, GPS data, and the fused position with the method presented in this paper. All three trajectories are plot against the real traveled path. As expected, the error on the position estimate is large for odometry, but also for GPS due to loss of the signal previous to finish the designed path. When fusing information from both sensors the position estimates for the designed path are better. Figure 2 shows a comparison between the fused position when using the presented method and when using EKF. As seen from the figure, slightly better results are found with the presented method, especially when GPS is lost, because we do not consider the sensors to be independent. Errors in the final $(x, y)$ position are $(0.81,0.54) \mathrm{m}$ for $\mathrm{EKF}$ and $(0.64$, $0.41) \mathrm{m}$ for the proposed method.

Figures 3 and 4 show the estimated uncertainty for the fused position estimates using the method we are proposing. The obtained uncertainties for the $(x, y)$ coordinates using the method presented in this paper are higher than the ones obtained when an EKF is used for the purpose of estimating the pose error. This is mainly due to two factors: first, the linearity on the uncertainty computation EKF assumes, and second, sensor independence as EKF does not consider cross-correlation between sensors. We have considered colored noise for the GPS, and cross-correlation between the current odometry pose and the previous GPS estimate as they both influence the actual fused pose. Our approach produces an uncertainty estimation which turns to be closer to the real measured errors. 


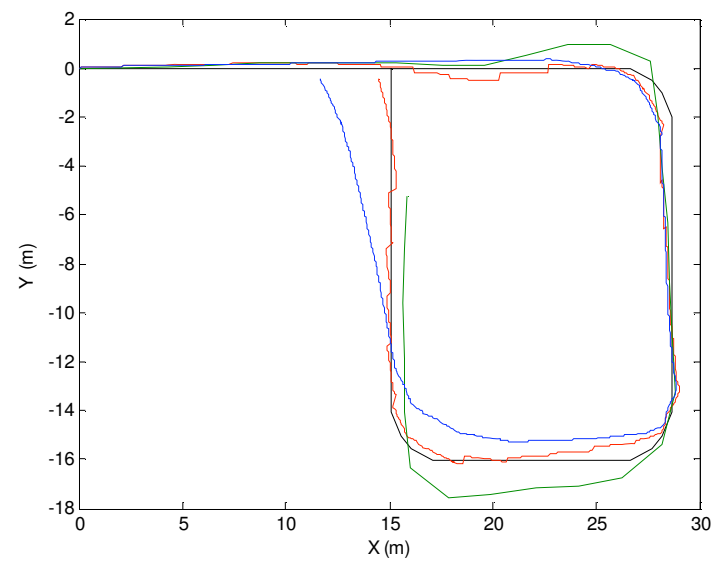

Figure 1 Odometry path (blue), GPS path (green) and fused path with presented method (red), versus real followed path (black).

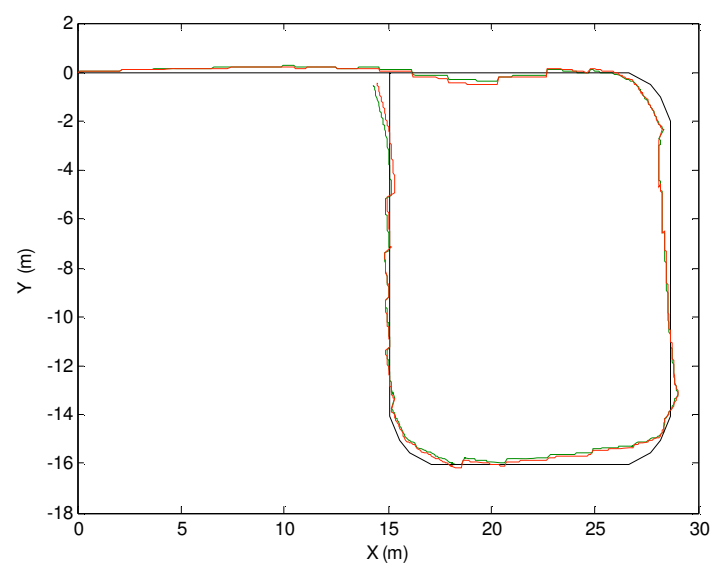

Figure 2 Fused trajectory, in meters, with the presented method (red) and the EKF (green) against desired path (black line).

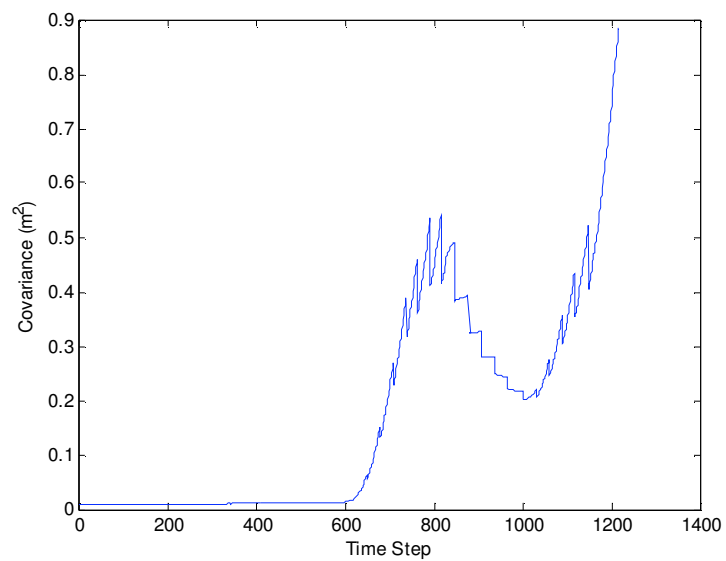

Figure 3 Fused position covariance estimate $\left(\mathrm{m}^{2}\right)$ for $x$ coordinate.

The computed uncertainty with the presented method does not depend on any parameter initialization as it is needed with the EKF, in this sense, we do not find variation of results depending on how the algorithm is initialized. It neither depends on the axis to which the robot is oriented. Obtained results are robust and consistent with the conducted experiment.

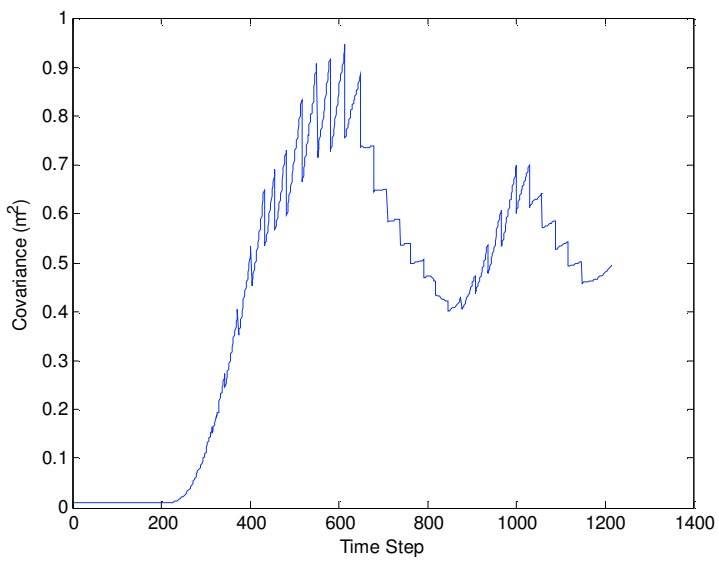

Figure 4 Fused position covariance estimate $\left(\mathrm{m}^{2}\right)$ for $y$ coordinate.

\section{CONCLUSIONS}

This paper has mainly focused on how to reduce the robot's position uncertainty by means of fusing information from different sensors using an accurately measure for the odometry position estimates uncertainty. The computation of the position uncertainty has been based on the research presented in previous works $[12,13]$, where a method to obtain an expression for the pose covariance matrix, when pose estimates are being obtained using internal sensors was proposed. We then dealt with the problem of reducing the uncertainty in the robot's pose estimate by using data from different sources. To this aim a data fusion technique based on probabilities that allow us using the previously derived odometry uncertainty formulation is proposed. The proposed method is particularized to the use of two pose estimates, DGPS (considering autocorrelated DGPS data) and odometry, and applied to a given kind of vehicle, consisting into a holonomic mobile robot architecture. Results of the fused position data are given together with covariance estimates for this position.

Although the presented method has been particularized to a given platform, it is general and valid for any number of sensors and robot architecture. Authors are actually working in the mathematical generalization of the data fusion technique presented when data from $N$ different sources are available. Some problems were found when dealing with bad DGPS readings due to multi-path, occlusions or loss of the differential corrections in the streets nearby to high buildings. Although an error estimate for the DGPS position is being maintained in the presented formulation, we found that when a DGPS position estimate arrives which is affected by one or some of these problems, it may have a low error estimate, although the position is far from the followed path. Due to this low error of the DGPS data, it is fused with the other sensor readings creating discontinuities in the followed path. We are, at present, working on different filter techniques to override this problem. 


\section{APPENDICES}

\section{A. USED PlatForm}

A standard Pioneer P3 AT robot named Helena, see figure 5, has been used in this study. The vehicle is electrically powered and it performs trajectories totally autonomously. Used internal sensors are, for the case at hands, two odometers attached to the shafts of the right and left drive wheel motors respectively.

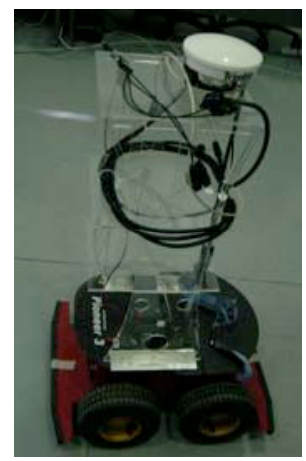

Figure 5 Pioneer P3 AT robot Helena used in this study.

The used driving architecture is differential. The kinematic model being used is found in any basic robotics book as [16]. Equation (15) expresses the kinematic model used for this study, where parameter $b$ is the robot width and $\left(V_{r}, V_{l}\right)$ denotes the right and left linear wheel control velocities respectively. The proposed model has the essential elements for the analysis and should be enough for control purposes

$$
\left(\begin{array}{l}
\dot{x} \\
\dot{y} \\
\dot{\theta}
\end{array}\right)=\left(\begin{array}{ll}
\frac{1}{2} \cos \theta & \frac{1}{2} \cos \theta \\
\frac{1}{2} \sin \theta & \frac{1}{2} \sin \theta \\
\frac{1}{b} & -\frac{1}{b}
\end{array}\right)\left(\begin{array}{l}
V_{r} \\
V_{l}
\end{array}\right)
$$

\section{B. Particularization of the UnCertainty COVARiance MATRICES FOR THE CONSIDERED ROBOT}

The first element in equation (2) is calculated as follows:

$$
\begin{aligned}
E\left[\Delta x_{k}^{\text {od }} \Delta x_{k}^{\text {od }} T\right]= & E\left[\frac{\Delta \hat{d}_{k}^{\text {right }}+\Delta \hat{d}_{k}^{\text {left }}}{2} \cos \left(\hat{\theta}_{k-1}\right) \frac{\Delta \hat{d}_{k}^{\text {right }}+\Delta \hat{d}_{k}^{\text {left }}}{2} \cos \left(\hat{\theta}_{k-1}\right)\right] \\
& =E\left[\left(\frac{\Delta \hat{d}_{k}^{\text {right }}+\Delta \hat{d}_{k}^{\text {left }}}{2} \cos \left(\hat{\theta}_{k-1}\right)\right)^{2}\right] \\
& =E\left[\left(\Delta d_{k}^{\text {right }}+\varepsilon^{\text {right }}+\Delta d_{k}^{l e f t}+\varepsilon^{l e f f}\right)^{2} \cos ^{2}\left(\hat{\theta}_{k-1}\right)\right] \\
& =\cdots=\left(\frac{1}{2}+\frac{1}{2} \cos \left(2 \theta_{k-1}\right) e^{-2 \sigma_{k-1}^{2}}\right) \\
& \cdot\left\{\left(\Delta d_{k}^{r i g h t}\right)^{2}+\left(\Delta d_{k}^{\text {left }}\right)^{2}+2 \Delta d_{k}^{\text {right }} \Delta d_{k}^{\text {left }}+\sigma_{r i g h t}^{2}+\sigma_{\text {left }}^{2}\right\}
\end{aligned}
$$

Two main assumptions have been made here. First, the orientation of the robot for time $k-1$ is considered as the sum of a deterministic angle and a random error in the form of $\hat{\theta}_{k-1}=\theta_{k-1}+\varepsilon_{\theta_{k-1}}$. Second, measures from both odometers have been considered independent. Defining the terms $k_{l}$ to $k_{5}$ as follows:

$$
\begin{aligned}
& k_{1}=\left(\Delta d_{k}^{\text {right }}\right)^{2}+\left(\Delta d_{k}^{\text {left }}\right)^{2} \\
& k_{2}=\left(\Delta d_{k}^{\text {right }}\right)^{2}-\left(\Delta d_{k}^{\text {left }}\right)^{2} \\
& k_{3}=\Delta d_{k}^{\text {right }} \Delta d_{k}^{\text {left }} \quad(19) \\
& k_{4}=\sigma_{\text {right }}^{2}+\sigma_{\text {left }}^{2}(20) \\
& k_{5}=\sigma_{\text {right }}^{2}-\sigma_{\text {left }}^{2}
\end{aligned}
$$

The rest of the elements in (2) are derived accordingly:

$$
\begin{aligned}
& E\left[\Delta x_{k}^{o d} \Delta x_{k}^{o d} T\right]=\frac{k_{1}+2 k_{3}+k_{4}}{4}\left(\frac{1}{2}+\frac{1}{2} \cos \left(2 \theta_{k-1}\right) \mathrm{e}^{-2 \sigma_{k-1}^{2}}\right) \\
& E\left[\Delta y_{k}^{o d} \Delta x_{k}^{o d^{T}}\right]=E\left[\Delta x_{k}^{o d} \Delta y_{k}^{o d^{T}}\right]=\frac{k_{1}+2 k_{3}+k_{4}}{4}\left(\frac{1}{2} \sin \left(2 \theta_{k-1}\right) \mathrm{e}^{-2 \sigma_{\sigma_{k-1}^{2}}^{2}}\right) \\
& E\left[\Delta x_{k}^{o d} \Delta \theta_{k}^{o d T}\right]=E\left[\Delta \theta_{k}^{o d} \Delta x_{k}^{o d T}\right]=\frac{k_{2}+k_{5}}{2 b} \cos \left(\theta_{k-1}\right) \mathrm{e}^{-\frac{1}{2} \sigma_{\theta_{k-1}}^{2}} \\
& E\left[\Delta y_{k}^{o d} \Delta y_{k}^{o d}\right]=\frac{k_{1}+2 k_{3}+k_{4}}{4}\left(\frac{1}{2}-\frac{1}{2} \cos \left(2 \theta_{k-1}\right) \mathrm{e}^{-2 \sigma_{k-1}^{2}}\right) \\
& E\left[\Delta y_{k}^{o d} \Delta \theta_{k}^{o d^{T}}\right]=E\left[\Delta \theta_{k}^{o d} \Delta y_{k}^{o d T}\right]=\frac{k_{2}+k_{5}}{2 b} \sin \left(\theta_{k-1}\right) \mathrm{e}^{-\frac{1}{2} \sigma_{\theta_{k-1}}^{2}} \\
& E\left[\Delta \theta_{k}^{\text {od }} \Delta \theta_{k}^{\text {od }}{ }^{T}\right]=\frac{k_{1}-2 k_{3}+k_{4}}{b^{2}}
\end{aligned}
$$

The second matrix, (3), is computed from the vector product $_{E}\left[\Delta P_{k}^{o d}\right]_{E}\left[\Delta P_{k}^{\text {od }}{ }^{T}\right]$, where,

$$
E\left[\Delta P_{k}^{\text {od }}\right]=\left(\begin{array}{c}
\frac{\Delta d_{k}^{\text {right }}+\Delta d_{k}^{\text {left }}}{2} \cos \left(\theta_{k-1}\right) \mathrm{e}^{-\frac{1}{2} \sigma_{\theta_{k-1}^{2}}^{2}} \\
\frac{\Delta d_{k}^{\text {right }}+\Delta d_{k}^{\text {left }}}{2} \sin \left(\theta_{k-1}\right) \mathrm{e}^{-\frac{1}{2} \sigma_{\theta_{k-1}}^{2}} \\
\frac{\Delta d_{k}^{\text {right }}-\Delta d_{k}^{\text {left }}}{b}
\end{array}\right)
$$

The full expression for this second matrix is not included, it is easily and directly derived from multiplying $E\left[\Delta P_{k}^{o d}\right] E\left[\Delta P_{k}^{o d^{T}}\right]$. The expression for the uncertainty of pose odometry increments is obtained by subtracting the two computed matrices: $E\left[\Delta P_{k}^{o d} \Delta P_{k}^{o d d^{T}}\right]-E\left[\Delta P_{k}^{o d}\right] E\left[\Delta P_{k}^{o d} T^{T}\right]$.

The total pose uncertainty needs, however, from the cross-covariance terms. Its computation is quite complicated as it has been outlined in section II and will not be included here. For a deeper mathematical insight, please refer to [12] and [17]. 


\section{UNCERTAINTY COVARIANCE MATRICES FOR GPS POSE ESTIMATES}

In order to obtain a fused pose estimate using the information from both, the DGPS and the odometry system, the covariance matrices $\operatorname{cov}\left[P_{k}^{G P S}\right]$ and $\operatorname{cov}_{k}^{o d-G P S}$ should be estimated, $\operatorname{cov}_{k}^{\text {od-GPS }}$ quantifies the dependency between odometry an DGPS. Both matrices shall be derived from experimental data. The first covariance matrix, $\operatorname{cov}\left[P_{k}^{G P S}\right]$, represents the uncertainty of the DGPS sensor at time $k$. A first experiment has been carried out gathering GPS position estimates of a fixed position during a period of 24 hours. Latitude, longitude and heading information were saved. Assuming that the mean position is the real position, the error vectors are computed subtracting the mean value of each component. For $k=0$ the DGPS covariance matrix $\operatorname{cov}\left[P_{k}^{G P S}\right]=E\left[e_{k}^{G P S}\left(e_{k}^{G P S}\right)^{\mathrm{T}}\right]$ is:

$$
\operatorname{cov}\left[P_{k=0}^{G P S}\right]=\left(\begin{array}{ccc}
1.217 & 0.96256 & 0 \\
0.96256 & 2.8346 & 0 \\
0 & 0 & 6.475
\end{array}\right)
$$

Computing the autocorrelation for each of the error vectors, using a window of 10 minutes (360 data points), we found that for latitude and longitude the hypothesis of white noise cannot be assumed. The case is different for the heading information where no autocorrelation with past data is found. So we consider colored noise for latitude and longitude:

$$
\begin{aligned}
& e_{k}^{G P S}=e_{k}^{G P S_{-} \text {color }}+e_{k}^{G P S_{-} \text {white }}=\operatorname{cov}^{G P S_{-} \text {color }} e_{k-1}^{G P S}+e_{k}^{G P S_{-} \text {white }} \\
& \operatorname{cov}\left[P_{k}^{G P S}\right]=E\left[e_{k}^{G P S}\left(e_{k}^{G P S}\right)^{T}\right]=\ldots= \\
& \cdot \operatorname{cov}^{G P S_{-} \text {color }} \operatorname{cov}\left[P_{k-1}^{G P S}\right] \operatorname{cov}^{G P S_{-} \text {colour }}{ }^{T}+\operatorname{cov}^{G P S_{-} \text {white }}
\end{aligned}
$$

Given the error vector for $k$ and $k-1$ and using ordinary least squares (OLS) estimation we obtain the matrix $\operatorname{cov}^{G P S_{-} c o l o r}$ and the vector $e_{k}^{G P S_{-} \text {white }}$ from which the matrix $\operatorname{cov}^{G P S}$ - white is determined,

$$
\begin{aligned}
\operatorname{cov}^{G P S} S_{-} \text {color } & =\left(\begin{array}{ccc}
1.0000011 & 0.00001424 & 0 \\
0.00001424 & 0.999976 & 0 \\
0 & 0 & 0
\end{array}\right) \\
\operatorname{cov}^{G P S} S_{- \text {white }} & =\left(\begin{array}{ccc}
1.83344 & 0.10481 & 0 \\
0.10481 & 3.26267 & 0 \\
0 & 0 & 647500
\end{array}\right) 10^{-5}(33)
\end{aligned}
$$

Another experiment was required in order to determine the matrix $\operatorname{cov}_{k}^{o d-G P S}$ expressing the correlation between GPS and odometry. The robot was manually driven along a
12 meters long straight line. Twelve consecutive trajectories were made. DGPS start and final points as well as odometry readings were saved. The error vectors, for $\Delta x, \Delta y$, and $\Delta \theta$, both, from the DGPS and odometry estimates, were computed subtracting the mean of the distances to the obtained vector of measures. The following matrix was found:

$\operatorname{cov}^{o d-G P S}=\left(\begin{array}{ccc}1.00462 & -0.69929 & 5.8597 \\ -0.69929 & 1.22223 & -5.53312 \\ 5.8597 & -5.53312 & 37.13316\end{array}\right)$

Just as a final note, the employed DGPS has been Trimble Agps106 model.

\section{REFERENCES}

[1] Ollero, A., "Robótica y Manipuladores Móviles”, Ed. Alfa Omega, 2000

[2] Dudek, G. and Jenkin, M., "Computational Principles of Mobile Robotics", Cambridge University Press, 2000.

[3] Gutiérrez, J., "Configuration and Construction of an Autonomous Vehicle for Tunnel Profiling Tasks", ITESM, Ph. D. Thesis, 2004.

[4] Rodríguez, J. et. al., "Automation of a Industrial Fork Lift Truck, Guided by Artificial Vision in Open Environments", Autonomous Robot, Vol. 5, No. 2, pp. 215-231, May, 1998.

[5] Stevenson, S.M., "Mars Pathfinder Rover-Lewis Research Center technology experiments program", Energy Conversion Engineering Conference (IECEC), Vol.1, Iss., 27 Jul-1 Aug 1997 Pages:722-727

[6] Leonard, J. J, and Durrant-Whyte, H. F., "Directed sonar sensing for mobile robot navigation", Kluwer Academic Publishers, 1992.

[7] Martinelli, A., "Modeling and Estimating the Odometry Error of a Mobile Robot." In Proceedings of the International Conference. on Nonlinear Control ( NOLCOS01), St Petesburg, July, 2001.

[8] Kelly A., "Linearized error propagation in odometry", Int. J. of robotics research, 23(2), Feb. 2004, pp. 179-218.

[9] Becker, J.C.; Simon, A., "Sensor and navigation data fusion for an autonomous vehicle" Proceedings of the IEEE Intelligent Vehicles Symposium (IV 2000), 2000, pages:156-161

[10] Durrant-Whyte, H.F. et. Al. "Position Estimation and Tracking Using Optical Range Data", Proceedings IEEE International Conference on Robotics and Automation (ICRA), Vol. 3, 1993, pp. 2172-2177.

[11] Bar-Shalom Y., X. Rong Li, T. Kirubarajan, "Estimation with Applications to Tracking and Navigation", John Wiley \& Sons,. 2001.

[12] Mirats-Tur, J.M., J.L Gordillo,. C. Albores,, "A Closed Form Expression for the Uncertainty in Odometry Position Estimate of an Autonomous Vehicle", Transactions on Robotics, 21(5), pp. 1017-1022, Oct. 2005.

[13] Mirats-Tur, J.M., J.L. Gordillo, C. Albores, "Communication on A Closed Form Expression for the Uncertainty in Odometry Position Estimate of an Autonomous Vehicle.", accepted to Transactions on Robotics, June 2007.

[14] A. Pozo-Ruz, "Sistema sensorial para localización de vehículos en exteriores", Ph.D. dissertation, Dept. Elect. Eng., University of Málaga, Spain, ISBN:84-699-8140-4, 2001.

[15] Scheding S., G. Dissanayake, EM Nebot, H. Durrant-Whyte, "An experiment in autonomous navigation of an underground mining vehicle", Transactions on Robotics and Automation, 15(1), 1999.

[16] A. Ollero, "Robótica: manipuladores y robots móviles", Ed. Marcombo Boixareu, Barcelona, 2001.

[17] Albores Borja, C., ""Analysis, Architecture, and Fusion Methods for Vehicle Automation", PhD thesis, ITESM, Monterrey, Nuevo Leon, Mexico, March 2007. 